\title{
Terminal Control Area Aircraft Scheduling and Trajectory Optimization Approaches
}

\author{
Marcella Samà ${ }^{1, \star \star \star}$, Konstantin Palagachev ${ }^{2}$, Andrea D’Ariano ${ }^{1}$, Matthias Gerdts $^{2}$, and Dario \\ Pacciarelli ${ }^{1}$ \\ ${ }^{1}$ Roma Tre University, Department of Engineering, \\ Section of Computer Science and Automation, \\ Via della Vasca Navale, 79, 00146 Rome, Italy \\ ${ }^{2}$ Universität der Bundeswehr München, Fakultät für Luft- und Raumfahrttechnik, \\ Institut für Mathematik und Rechneranwendung, \\ Werner-Heisenberg-Weg 39, D-85577 Neubiberg/München, Germany
}

\begin{abstract}
Aviation authorities are seeking optimization methods to better use the available infrastructure and better manage aircraft movements. This paper deals with the realtime scheduling of take-off and landing aircraft at a busy terminal control area and with the optimization of aircraft trajectories during the landing procedures. The first problem aims to reduce the propagation of delays, while the second problem aims to either minimize the travel time or reduce the fuel consumption. Both problems are particularly complex, since the first one is NP-hard while the second one is nonlinear and a combined solution needs to be computed in a short-time during operations. This paper proposes a framework for the lexicographic optimization of the two problems. Computational experiments are performed for the Milano Malpensa airport and show the existing gaps between the performance indicators of the two problems when different lexicographic optimization approaches are considered.
\end{abstract}

\section{Introduction}

Air traffic control must ensure safe, ordered and rapid transit of aircraft on the ground and in the air segments. Airports have become the major bottleneck in air traffic control due to the strong increase in air traffic demand of the last decades. Operations in air traffic control are performed by human controllers aided by some computer support, often limited to a graphical representation of the aircraft position and speed. The controllers of the Terminal Control Area (TCA) determine feasible plans by selecting the order in which the different aircraft perform their operations and the aircraft timing in each airport resource. Usually the controllers start from a solution computed using some scheduling rule, e.g. the First Come First Served (FCFS) rule, and make then adjustments to improve the schedule. However, the resulting solution does not fully take into consideration neither the propagation of aircraft delays nor the minimization of other relevant performance indicators [16].

\footnotetext{
^e-mail: sama@ing.uniroma3.it

${ }^{\star}$ Acknowledgement: The corresponding author was supported by the German academic exchange service (DAAD) with a Research Grant for PhD candidates, Funding Program Number 57130097
} 
This paper deals with the Terminal Control Area Aircraft Scheduling Problem (TCA-ASP) and the Aircraft Trajectory Optimization Problem (ATOP) for landing operations in a busy TCA. The first problem is to compute a conflict-free schedule for all aircraft with the minimization of the overall aircraft delays. The second problem is to compute a landing trajectory for each aircraft with either the minimization of the final time or the minimization of the fuel consumption. The trajectory with minimal travel time helps to reduce the individual aircraft delay, while trajectories with minimal fuel consumption can increase aircraft delays. According to recent surveys on the aircraft scheduling literature $[1-3,8,11]$, there is still a need to develop integrated optimization approaches for solving the TCA-ASP and the ATOP. This topic requires to take simultaneously into consideration the following aspects:

1. In the TCA-ASP, the optimization approach should be able to incorporate all the information that ensures that the aircraft schedule is compliant with the safety regulations. In most of the existing approaches, the characteristics of the airport infrastructure are drastically simplified and the flight paths are aggregated. Thus potential aircraft conflicts may not be detected and solved at the level of runways, ground and air segments of the TCA.

2. In the ATOP, the trajectory optimization approach should be done in order to ensure safety and efficiency of the flight. A detailed approach for planning the flying time of each aircraft should take into account the behaviour of all aircrafts that share the same TCA resources.

3. The available time for developing a new schedule for the integrated problem can be very limited, since a computerized scheduler should be able to promptly react to any change occurring in the TCA.

This paper addresses the first aspect by formulating the TCA-ASP as a job shop scheduling model with additional constraints. This modelling approach is able to consider the airspace interactions between aircraft in each TCA resource. However, a pre-defined trajectory has to be modelled for each aircraft. The trajectory can be fine-tuned before/after solving the TCA-ASP.

The second aspect is addressed by formulating and solving an optimal control problem, whose solution provides the reference trajectory for each aircraft flying through each resource.

The third aspect requires to use fast and effective approaches to solve the TCA-ASP and the ATOP, and for integrating their solutions. In this paper, we solve the TCA-ASP via AGLIBRARY, a dedicated software developed by Roma Tre University, and we compute a near-optimal solution to the ATOP of each landing aircraft via $O C P I D-D A E 1$, a dedicated software developed by the University of the Federal Armed Forces at Munich. The integration of the TCA-ASP and ATOP is obtained via a new optimization framework developed in this paper.

The remaining of this paper is organized as follows. Section 2 gives a brief description of the TCA-ASP models and algorithms of AGLIBRARY, Section 3 describes the approach used in OCPIDDAE1 to solve the ATOP, Section 4 presents the new optimization framework for integrating the TCA-ASP and ATOP solutions. The integrated solution is obtained by a lexicographic optimization, in which the TCA-ASP and ATOP are solved sequentially. We study various settings of the framework in which we change the order in which the two optimization problems are solved and the performance indicators to be optimized. Section 5 concludes the paper and outlines further research directions.

\section{Terminal Area Aircraft Scheduling Approach}

Let us consider a set of landing and take-off aircraft to be scheduled in the TCA. For each aircraft, a feasible trajectory in the TCA is provided together with its current position, and its scheduled occupancy times in the required TCA resources to accomplish the arriving/departing procedures. The 
TCA-ASP is to assign the start time to each aircraft in each TCA resource it crosses in such a way that all the potential conflict situations between aircraft are solved, the safety constraints are respected and the propagation of aircraft delays is minimized.

To model the TCA-ASP, we follow the approach of Bianco et al. [4], based on the no-wait version of the job shop scheduling problem. However, this paper is based on the alternative graph model introduced by Mascis and Pacciarelli [9]. The alternative graph model is able to model with higher precision some relevant TCA-ASP aspects such as modeling the airborne holding, the aircraft trajectory in each TCA resource, the hosting of multiple aircraft simultaneously in each air segment, and the hosting of individual aircraft simultaneously in each runway.

We now formalize TCA-ASP model. An operation $i$ denotes the traversing of a TCA resource (i.e. holding circle, air segment, runway) by a job (i.e. aircraft). The timing of an operation $i$ represents the aircraft trajectory in the corresponding resource. Given the timing related to each aircraft operation, the associated schedule is conflict-free if the minimum time separation constraints are satisfied on each resource.

Let $G=(N, F, A)$ be the alternative graph composed of the following sets: $N=\{0,1, \ldots, n\}$ is the set of nodes, where nodes 0 and $n$ represent the start and the end operations of the schedule, while the other nodes are related to the start of the other operations; $F$ is the set of fixed directed arcs that model the sequence of operations to be executed by each aircraft; $A$ is the set of alternative pairs that model the sequencing decision and inter-aircraft safety separations. Each pair is composed of two alternative directed arcs.

Let $t_{i}$ be the start time of operation $i$, each node $i \in N$ of the alternative graph is associated with $t_{i}$, and corresponds to the entrance of the associated aircraft in the associated resource. By definition, the start time of the schedule is a known value, e.g. $t_{0}=0$, and the end time of the schedule is a variable $t_{n}$.

Let $\sigma(i)$ denote the operation following $i$. Each fixed directed $\operatorname{arc}(i, \sigma(i)) \in F((\sigma(i), i) \in F)$ has a weight $w_{i \sigma(i)}^{F}\left(w_{\sigma(i) i}^{F}\right)$, i.e. the constraint $t_{\sigma(i)} \geq t_{i}+w_{i \sigma(i)}^{F}\left(t_{i} \geq t_{\sigma(i)}+w_{\sigma(i) i}^{F}\right)$. The fixed arc weight $w_{i \sigma(i)}^{F}$ $\left(-w_{\sigma(i) i}^{F}\right)$ models the minimum (maximum) traversing time between the start of $i$ and the start of $\sigma(i)$.

Each alternative pair $((i, j),(h, k)) \in A$ has two arcs with weight $w_{i j}^{A}$ and $w_{h k}^{A}$. The alternative arc weight $w_{i j}^{A}$ represents a minimum separation time between the start of $i$ and the start $j$. In particular, $w_{i j}^{A}\left(w_{h k}^{A}\right)$ can be sequence-dependent, when nodes $i$ and $j(h$ and $k$ ) are operations of different jobs. Also, there can be multiple alternative arcs between nodes $i$ and $j$.

A selection $S$ is a set of alternative arcs, at most one from each pair. Given the alternative pair $((i, j),(h, k)) \in A$, we select either the $\operatorname{arc}(i, j)$ or the $\operatorname{arc}(h, k)$, i.e. either the constraint $t_{j} \geq t_{i}+w_{i j}^{A}$ or the constraint $t_{k} \geq t_{h}+w_{h k}^{A}$.

An ASP solution is a complete selection $S^{c}$, where an arc for each alternative pair of the set $A$ is selected and the connected graph $\left(N, F, S^{c}\right)$ has no positive weight cycles. Note that a positive weight cycle represents an operation preceding itself, which is an infeasibility. Given a feasible schedule $S^{c}$, $t_{i}$ is the length of a longest path from 0 to $i\left(l^{S^{c}}(0, i)\right)$. A fixed $\operatorname{arc}(k, n)$ between the end node $k$ of each job and node $n$ is added to the alternative graph, with weight equal to the scheduled arrival time at the runway with negative sign. A selection $S^{c}$ is optimal if $l^{S^{c}}(0, n)$ is minimum over all the solutions. In this work, we minimize the maximum tardiness, that is $l^{S^{c}}(0, n)$.

We compute a near-optimal solution to the proposed TCA-ASP formulation via a branch $\&$ bound algorithm [6] truncated after a maximum computation time (i.e. after 180 seconds), implemented in the AGLIBRARY solver. A more detailed description of the alternative graph models, algorithms and solver can be found in D'Ariano et al. [5, 6] and Samà et al. [13-15]. 


\section{Aircraft Trajectory Optimization Approach}

The ATOP is an optimal control problem, representing a simplified, two-dimensional point mass model of the aircraft's dynamics. It includes the state variables $x, h, V$ and $\gamma$, representing respectively the aircraft's position, altitude, speed and climb angle. The control inputs to the model are given by the angle of attack $\alpha$ and the thrust level position $\delta_{T}$, constrained in the interval [0,1]. Hence the actual thrust applied to the aircraft is given by $T=T_{\max } \delta_{T}$, where $T_{\max }$ is the maximum thrust available. The list $L$ and drag $D$ forces, acting on the aircraft, are given by

$$
L=\frac{\rho}{2} \cdot V^{2} \cdot S_{r e f} \cdot C_{L} \quad \text { and } \quad D=\frac{\rho}{2} \cdot V^{2} \cdot S_{r e f} \cdot C_{D}
$$

where $C_{L}$ and $C_{D}$ are respectively the lift and drag coefficients

$$
C_{L}=C_{L_{0}}+C_{L_{\alpha}} \alpha \quad \text { and } \quad C_{D}=C_{D_{0}}+C_{D_{2}} C_{L}^{2}
$$

Note that $C_{L_{0}}, C_{L_{\alpha}}, C_{D_{0}}$ and $C_{D_{2}}$ are unit-less parameters, depending on the flat's configuration of the aircraft, as well as on the aircraft itself. Furthermore, $S_{\text {ref }}$ is the aircraft's wing reference surface, $m$ is the aircraft's mass, $\rho$ is the air density, while $g$ is the gravitational acceleration. Hence ATOP reads as follow

$$
\begin{array}{ll}
\text { Minimize } & \omega \cdot t_{f}+(1-\omega) \int_{0}^{t_{f}}\left\|\delta_{T}(t)\right\|^{2} d t \\
\text { subject to } & \dot{x}(t)=V(t) \cdot \cos \gamma(t) \\
\dot{h}(t) & =V(t) \cdot \sin \gamma(t) \\
& \dot{V}(t)=\frac{T(t)-D(t)}{m}-g \cdot \sin \gamma(t) \\
& \dot{\gamma}(t)=\frac{L(t)-m \cdot g \cdot \cos \gamma(t)}{m \cdot V(t)} \\
& (x, h, V, \gamma)(0)=\left(x_{0}, h_{0}, V_{0}, \gamma_{0}\right) \\
& (x, h, V, \gamma)\left(t_{f}\right)=\left(x_{f}, h_{f}, V_{f}, \gamma_{f}\right) .
\end{array}
$$

Note that in (1) the parameter $\omega$ is responsible for the minimum travel time problem $(\omega=1$, ATOP-tt), the minimum fuel consumption problem $(\omega=0$, ATOP-fc) or any combination of the two problems $(\omega \in(0,1))$. Furthermore, equations (6)-(7) are responsible for fixing the states of the problem at initial and final time (depending on the resource through which the aircraft is flying). We emphasize that for sake of simplicity, we are not including any bounds for the state variables, as well as any constraint in the formulation of ATOP. For more precise formulation, we refer the interested reader to $[10,12]$.

In order to compute the solution of ATOP, we adopt a standard technique in optimal control theory, known as discretize-then-optimize. It consists in the approximation of the original infinite dimensional problem by a suitable finite dimensional one. Then, the approximated problem is solved and the solution is interpreted as an approximation of the solution of the original problem (see [7] for more details). One of the main advantages is that the optimal solution of ATOP can be reached with high precision and its optimality is guaranteed by mathematical theory. Furthermore, the optimal solution is easily recomputable, once the dynamics or the cost function are perturbed.

\section{Integrated Approaches}

The problems studied in the previous sections are now combined in order to find an integrated solution. To this aim, we use the framework of Figure 1. 


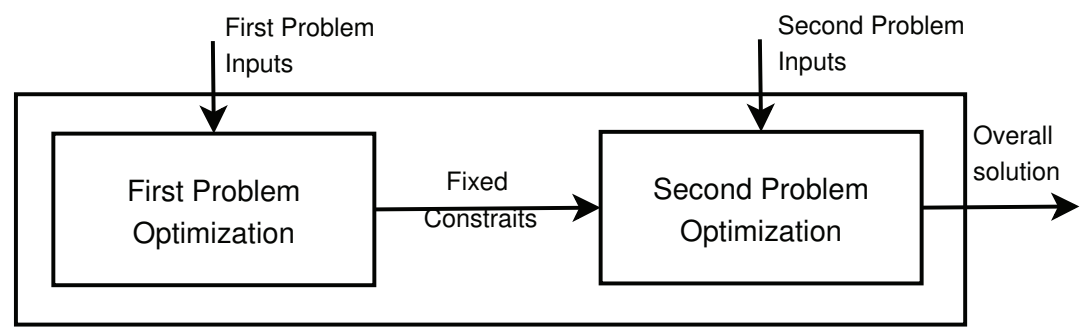

Figure 1. Solution framework

The idea is to solve the two problems sequentially and to define a lexicographic order of importance for the performance indicators to be optimized. Therefore, the following alternative integrated approaches are investigated:

- First ATOP, then TCA-ASP: We first compute the near-optimal trajectory of each landing aircraft in each TCA resource it requires. This trajectory is found by either minimizing the aircraft travel time (ATOP-tt) or the fuel consumption (ATOP-fc), and it is used to form the job in the alternative graph corresponding to the investigated aircraft. In this integrated approach, we need to set a fixed travel time for the aircraft in the TCA-ASP problem. This is modelled by setting the weight $w_{i \sigma(i)}^{F}$ of each fixed arc $(i, \sigma(i))$ equal to the corresponding traversing time in the ATOP, and $w_{\sigma(i) i}^{F}=-w_{i \sigma(i)}^{F}$.

- First TCA-ASP, then ATOP: We first compute the near-optimal solution to the TCA-ASP. The resulting connected graph $\left(N, F, S^{c}\right)$ is then studied in order to find a range of feasible values for the travel time of each landing aircraft in each resource. Specifically, we set a minimum and a maximum value of the travel time such that the selection $S^{c}$ is respected in any feasible ATOP solution. Clearly, the ATOP optimization only focuses on the aircraft that offer some degree of flexibility, i.e. on the aircraft having a different value of the minimum and maximum travel times on at least one TCA resource.

\section{Computational Experiments}

Figure 2 shows the TCA scheme of Milano Malpensa airport (MXP). There are two interdependent runways (RWY 35L, RWY 35R), used both for departing and arriving procedures. The MXP resources are 3 airborne holding circles (resources 1-3, named TOR, MBR, SRN), 11 air segments for arriving procedures (resources 4-14), 1 common glide path (resource 15), and 2 runways (resources 16-17).

Table 1 describes the 15 instances that we generated with random entrance delays. Column 1 presents the TCA (FCO and MXP), Columns 2-3 the number of landing and take-off aircraft, Columns 4-5 the maximum and average entrance delays (in seconds). The entrance delays are randomly generated for the first half aircraft entering the TCA according to a given distribution (either negative exponential or Gaussian distributions). The exit delays are measured as a positive deviation from the scheduled take-off/landing time.

Table 1. Problem instances

\begin{tabular}{|c|c|c|c|c|}
\hline TCA & $\begin{array}{c}\text { Landing } \\
\text { Aircraft }\end{array}$ & $\begin{array}{c}\text { Take-off } \\
\text { Aircraft }\end{array}$ & $\begin{array}{c}\text { Max Entrance } \\
\text { Delay (sec) }\end{array}$ & $\begin{array}{c}\text { Avg Entrance } \\
\text { Delay (sec) }\end{array}$ \\
\hline MXP & 14 & 6 & 1792.9 & 869.6 \\
\hline
\end{tabular}




$\begin{array}{llll}\text { Holding } & \text { Landing } & \text { Common } & \text { Runways } \\ \text { Circles } & \text { Air Segments } & \text { Glide Path } & \end{array}$

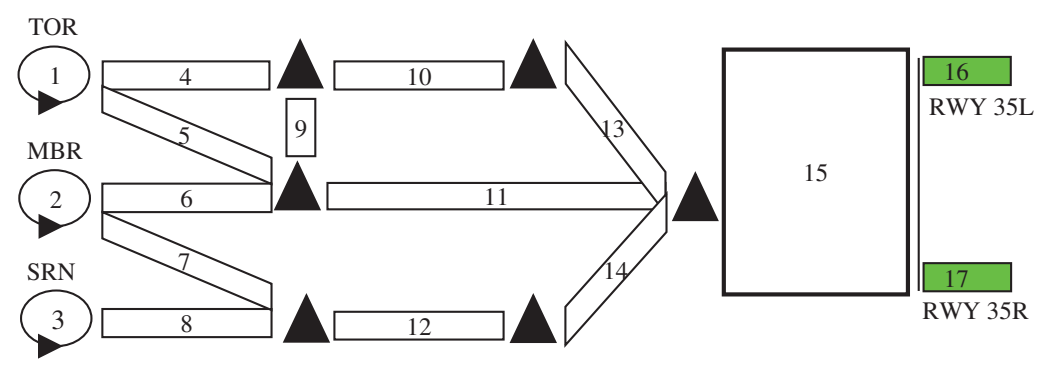

Figure 2. Milano Malpensa (MXP) Terminal Control Area

Preliminary computational experiments have been performed on a Quad-Core Intel Xeon E5 3.7 GHz processor with $32 \mathrm{~GB}$ RAM, under OS X 10.10.3. Table 2 presents an average comparison of the various integrated approaches on the 15 instances of Table 1 . Column 1 indicates the integrated approach configuration and Columns 2-4 the average value of a specific performance indicator. The best value for each column is reported in bold. The results show an interesting trade-off between the performance indicators related to the different problems. When minimizing the maximum tardiness and the travel time, the lexicographic order in which the two problems are tackled does not impact on the solution quality, suggesting a strong correlation between the two indicators. A different trend is observed when dealing with the minimization of the fuel consumption. The lexicographic order in which the two problems are tackled impacts the solution quality in terms of all performance indicators.

Table 2. Trade-off between the three performance indicators

\begin{tabular}{|c|c|c|c|}
\hline Integrated Approach & Max Tardiness & Travel Time & Fuel Consumption \\
\hline TCA-ASP/ATOP-tt & $\mathbf{1 4 9 4 . 3}$ & $\mathbf{9 8 1 2 . 0}$ & 2369.9 \\
TCA-ASP/ATOP-fc & $\mathbf{1 4 9 4 . 3}$ & 12188.8 & 756.4 \\
ATOP-tt/TCA-ASP & $\mathbf{1 4 9 4 . 3}$ & $\mathbf{9 8 1 2 . 0}$ & 2369.9 \\
ATOP-fc/TCA-ASP & 1729.2 & 13278.0 & $\mathbf{2 7 6 . 0}$ \\
\hline
\end{tabular}

\section{Conclusions and Further Research}

This paper addresses the problem of developing an integrated framework for the TCA-ASP and the ATOP. Various integrated approaches are investigated and tested on a practical case study. Preliminary results quantify the gap between the performance indicators, highlighting the multi-objective nature of the problem.

Further research should be dedicated at least to the following issues: evaluate the alternative framework on different instances, improve the effectiveness of the integrated approaches, validate the approaches at the traffic control center.

\section{References}

[1] Allahverdi, A., Ng, C.T., Cheng, T.C.E., Kovalyov, M.Y. (2008) A survey of scheduling problems with setup times or costs. European Journal of Operational Research 187 (3) 985-1032 
[2] Ball, M.O., Barnhart, C., Nemhauser, G., Odoni, A. (2007) Air Transportation: Irregular Operations and Control. In: G. Laporte and C. Barnhart (Eds.). Handbooks in Operations Research and Management Science 14 1-67

[3] Bennell, J.A., Mesgarpour, M., Potts, C.N. (2011) Airport runway scheduling. 4OR - Quarterly Journal of Operations Research 9 (2) 115-138

[4] Bianco, L., Dell'Olmo, P., Giordani, S. (2006) Scheduling models for air traffic control in terminal areas. Journal of Scheduling 9 (3) 180-197

[5] D’Ariano, A., Pistelli, M., Pacciarelli, D. (2012) Aircraft retiming and rerouting in vicinity of airports. IET Intelligent Transport Systems 6 (4) 433-443

[6] D’Ariano, A., Pacciarelli, D., Pistelli, M., Pranzo, M. (2015) Real-time scheduling of aircraft arrivals and departures in a terminal maneuvering area. Networks 65 (3) 212-227

[7] Gerdts, M. (2012) Optimal Control of ODE and DAEs, 458p. Walter de Gruyter GmbH \& Co.KG, Berlin Boston.

[8] Kuchar, J.K., Yang, L.C. (2000) A Review of Conflict Detection and Resolution Modeling Methods. IEEE Trans. on Intelligent Transp. Systems 4 (1) 179-189

[9] Mascis, A., Pacciarelli, D. (2002) Job shop scheduling with blocking and no-wait constraints. European Journal of Operational Research 143 (3), 498-517

[10] Palagachev, K., Rieck, M., Gerdts, M. (2013) A Mixed-Integer Optimal Control Approach for Aircraft Landing Model. Proceedings of the 3rd International Conference on Models and Technology for Intelligent Transportation Systems (MT-ITS 2013), 10 p., Dresden, Germany

[11] Pellegrini, P., Rodriguez, J. (2013) Single European sky and single European railway area: A system level analysis of air and rail transportation. Transportation Research Part A 57 (1) 64-86

[12] Rieck, M., Richter, M., Holzapfel, F. (2013) Discrete Control Dependent Constraints in Multiple Shooting Optimal Control Problems. Proceedings of the AIAA Guidance, Navigation, and Control (GNC) Conference, Guidance, Navigation, and Control and Co-located Conferences (AIAA 20134526)

[13] Samà, M., D’Ariano, A., Pacciarelli, D. (2013) Rolling Horizon Approach for Aircraft Scheduling in the Terminal Control Area of Busy Airports. Transportation Research Part E 60 (1), 140155

[14] Samà, M., D’Ariano, A., D'Ariano, P. Pacciarelli, D. (2014) Optimal aircraft scheduling and routing at a terminal control area during disturbances. Transportation Research Part C 47 (1) $61-85$

[15] Samà, M., D’Ariano, A., D’Ariano, P. Pacciarelli, D. (2015) Air traffic optimization models for aircraft delay and travel time minimization in terminal control areas Public Transport 7 (3) 321-337

[16] Samà, M., D’Ariano, A., D’Ariano, P. Pacciarelli, D. (2016) Scheduling models for optimal aircraft traffic control at busy airports: Tardiness, priorities, equity and violations considerations OMEGA doi:10.1016/j.omega.2016.04.003 